IRRITABLE BOWEL SYNDROME

\title{
Food elimination based on $\lg G$ antibodies in irritable bowel syndrome: a randomised controlled trial
}

\author{
W Atkinson, T A Sheldon, N Shaath, P J Whorwell
}

Gut 2004;53:1459-1464. doi: 10.1136/gut.2003.037697

See end of article for authors' affiliations

.....................

Correspondence to: Dr P J Whorwell, Department of Medicine, University Hospital of South Manchester,

Manchester M20 2LR, UK; peter.whorwell@ smuht.nwest.nhs.uk

Revised version received 13 April 2004

Accepted for publication

13 April 2004
Background: Patients with irritable bowel syndrome (IBS) often feel they have some form of dietary intolerance and frequently try exclusion diets. Tests attempting to predict food sensitivity in IBS have been disappointing but none has utilised lgG antibodies.

Aims: To assess the therapeutic potential of dietary elimination based on the presence of $\lg G$ antibodies to food.

Patients: A total of 150 outpatients with IBS were randomised to receive, for three months, either a diet excluding all foods to which they had raised lgG antibodies (enzyme linked immunosorbant assay test) or a sham diet excluding the same number of foods but not those to which they had antibodies.

Methods: Primary outcome measures were change in IBS symptom severity and global rating scores. Noncolonic symptomatology, quality of life, and anxiety/depression were secondary outcomes. Intention to treat analysis was undertaken using a generalised linear model.

Results: After 12 weeks, the true diet resulted in a $10 \%$ greater reduction in symptom score than the sham diet (mean difference 39 (95\% confidence intervals (CI) $5-72$ ); $p=0.024$ ) with this value increasing to $26 \%$ in fully compliant patients (difference $98(95 \% \mathrm{Cl} 52-144) ; p<0.001)$. Global rating also significantly improved in the true diet group as a whole $(p=0.048, N N T=9)$ and even more in compliant patients $(p=0.006, N N T=2.5)$. All other outcomes showed trends favouring the true diet. Relaxing the diet led to a $24 \%$ greater deterioration in symptoms in those on the true diet (difference 52 ( $95 \% \mathrm{Cl} 18-88$ ); $\mathrm{p}=0.003$ ). Conclusion: Food elimination based on lgG antibodies may be effective in reducing IBS symptoms and is worthy of further biomedical research. $\mathrm{c}$ rritable bowel syndrome (IBS) is a common disorder which causes abdominal pain, abdominal distension, and bowel dysfunction, characterised by loose bowels, constipation, or a fluctuation between these two extremes. ${ }^{1}$ This condition significantly impairs quality of life and places a large burden on health care resources. ${ }^{2}$ Treatment of IBS is largely based on the use of antispasmodics, antidepressants, and medications that modify bowel habit, depending on whether constipation or diarrhoea is the predominant problem. ${ }^{1}$ The notorious inadequacies of current drug therapy lead to much patient dissatisfaction and a tendency for patients to seek a variety of alternative remedies, especially of a dietary nature.

IBS is likely to be a multifactorial condition involving a number of different mechanisms although the prominence of any particular factor may vary from patient to patient. ${ }^{13}$ However, patients often strongly believe that dietary intolerance significantly contributes to their symptomatology and some sufferers seem to benefit from eliminating certain foods from their diet. Detection of food intolerance is often difficult due to its uncertain aetiology, non-specific symptomatology, and relative inaccessibility of the affected organ. Thus most previous studies have relied on the use of exclusion diets, which are extremely labour intensive and time consuming. ${ }^{45}$ Attempts to "test" for food intolerance in IBS have largely focused on "classic" food allergy based on the presence of IgE mediated antibody responses, although it appears that these "immediate type" reactions are probably quite rare in this condition. ${ }^{6-10}$ It is therefore possible that adverse reactions to food in patients with IBS might be due to some other form of immunological mechanism, rather than dietary allergy. Such reactions could be mediated by IgG antibodies, which characteristically give a more delayed response following exposure to a particular antigen ${ }^{11}$ and have been implicated in some cases of food hypersensitivity. ${ }^{12-14}$ However, this mechanism is controversial and is considered by some to be physiological $^{15-17}$ especially as IgG food antibodies can be present in apparently healthy individuals. ${ }^{18-20}$ It has previously been suggested that IgG food antibodies may have a role in $\mathrm{IBS}^{21}$ and it was therefore the purpose of this study to formally evaluate, in a randomised controlled trial, the therapeutic potential of an elimination diet based on the presence of IgG antibodies to food in patients with IBS.

\section{PATIENTS AND METHODS}

\section{Patients}

All patients with uncomplicated IBS (all bowel habit subtypes) attending the Gastroenterology Department at the University Hospital of South Manchester were considered eligible for the study, and those aged between 18 and 75 years, who satisfied the Rome II criteria, ${ }^{22}$ were invited to participate. Tertiary care patients were excluded from the study. All patients had normal haematology, biochemistry, and endoscopic examination when indicated. Coeliac disease was excluded using the tissue transglutaminase test and a hydrogen breath test was used for excluding lactose intolerance. Patients were also excluded from participating in the study if they had any significant coexisting disease or a history of gastrointestinal surgery, excluding appendicectomy, cholecystectomy, and hiatus hernia repair. The study was approved by the local ethics committee and all patients provided written informed consent.

\section{Methods}

The study used a double blind, randomised, controlled, parallel design in which patients were randomised to either a "true" diet or a "sham" diet control group. At screening,

Abbreviations: IBS, irritable bowel syndrome; ELISA, enzyme linked immunosorbant assay; $A U$, arbitrary unit; $H A D$, hospital anxiety and depression scale; QOL, quality of life; NNT, number needed to treat 
blood was taken and sent, with only a numerical identifier, to YorkTest Laboratories Ltd (York, UK) where an enzyme linked immunosorbant assay (ELISA) test was performed to detect the presence of IgG antibodies specific to a panel of 29 different food antigens. This test has been described in detail elsewhere ${ }^{23}$ and involves specimens being diluted 1/50, 1/150, and $1 / 450$ with each dilution applied to an allergen panel. Each test was calibrated using 0 arbitrary unit (AU) and 25 AU standards prepared from a serum with a high IgG titre to a cow's milk allergen extract. A positive control serum at $45 \mathrm{AU}$ was applied to each test. The test results were obtained from the $1 / 150$ dilution of the specimen. Where a high specimen background was observed, the test results were obtained from the $1 / 450$ dilution. The threshold for a positive (reactive) result was selected as three times the background signal obtained by the same sample against a no food allergen coated control well equivalent to $3 \mathrm{AU}$. Test results were scored as positive or negative only, relative to this cut off.

Staff based at the YorkTest Laboratories produced a true and sham diet sheet for each patient. The sham diet eliminated the same number of foods to which a patient exhibited IgG antibodies but not those particular foods. The goal was to try and include in the sham diet an equally difficult to eliminate staple food for every staple food in the true diet. Thus cow's milk was (generally) replaced with potato, wheat with rice, and yeast with whole egg, where this was possible. Nut reactivities were replaced with other nuts in the sham diet, and legumes with other legumes, but this was not systematised.

The true and sham diet sheets for each patient were sent to the University of York, again with only a number for identification. Patients were allocated to one of the two diet sheets based on a randomisation schedule developed using a random computer number generator. Thus patients would receive either an elimination diet based on their true sensitivity results (true diet) or a sham diet. All patients and clinical staff in the Gastroenterology Research Department and YorkTest Laboratory were blinded to the group assignment of all patients for the duration of the study.

Patients were given their allocated diet sheet by staff at the Gastroenterology Research Department and asked to eliminate the indicated foods from their diet for a period of 12 weeks. They also received a booklet with advice on eliminating the different foods and the telephone contact details of a free nutritional advisor whom they were able to contact for further advice if necessary.

Symptoms were assessed using a questionnaire scoring system validated for use in IBS, including the IBS symptom severity score (range 0-500). ${ }^{24}$ This is a system for scoring pain, distension, bowel dysfunction, and general well being, with mild, moderate, and severe cases indicated by scores of $75-175,175-300$, and $>300$, respectively. A reduction in score of 50 or over is regarded as a clinically significant improvement. $^{24}$ Non-colonic symptomatology, ${ }^{25}$ such as lethargy, backache, nausea, and urinary symptoms, was assessed and scored using visual analogue scales (range 0500). Quality of life (QOL) was measured using an instrument proven to be sensitive to change in IBS (range 0-500). ${ }^{26-28}$ Anxiety and depression were evaluated using the hospital anxiety and depression scale (HAD). ${ }^{29}$ This instrument scores anxiety and depression up to a maximum score of 21 for each parameter, with a score above 9 indicating significant psychopathology. Data on these measures were recorded at baseline and after 4, 8, and 12 weeks of the dietary intervention period. In addition, at 4, 8, and 12 weeks, patients were asked to give a global rating of their IBS using the question, "Compared with your IBS before you started the food elimination diet, are you now: terrible, worse, slightly worse, no change, slightly better, better, or excellent?" The atopic status of all patients entering the study was also assessed.

During the treatment phase, patients were allowed to take concomitant medication provided it had been constant for six months prior to the start of the study. They were encouraged not to alter medication use during the course of the trial but any changes were recorded. Any patient withdrawing from the study was encouraged to complete a final symptom questionnaire at week 12 and their reasons for withdrawal were recorded. At the end of 12 weeks, patients were asked to resume consumption of the foods they had been advised to eliminate in order to assess the effect of their reintroduction. Patients were then reassessed after four weeks using the same measures and the result compared with their scores at the end of the elimination phase.

\section{Data analysis}

Questionnaires were scored by an assessor blinded to the randomisation. The primary outcome measures were changes in IBS symptom severity score and global impact score at 12 weeks. Changes in non-colonic symptoms, QOL, and HAD scores were regarded as secondary outcome measures. Two sample $t$ tests were used to establish whether there was an overall difference in the change in continuous outcome measures between the two groups of patients. Patients were analysed according to the group to which they were randomised, independent of their adherence to the diet. The global impact score, an ordered categorical variable, was analysed using a Wilcoxon Mann-Whitney test to compare the numbers in the active and sham groups showing significant improvement ("better" or "excellent"), no significant change ("slightly worse", "no change", or "slightly better"), and significant deterioration ("worse" or "terrible"). The number needed to treat (NNT) was calculated from the global impact score by calculating the reciprocal of the difference in probability of a significant improvement between the treatment and control groups. General linear modelling in SPSS was used to explore whether there was a

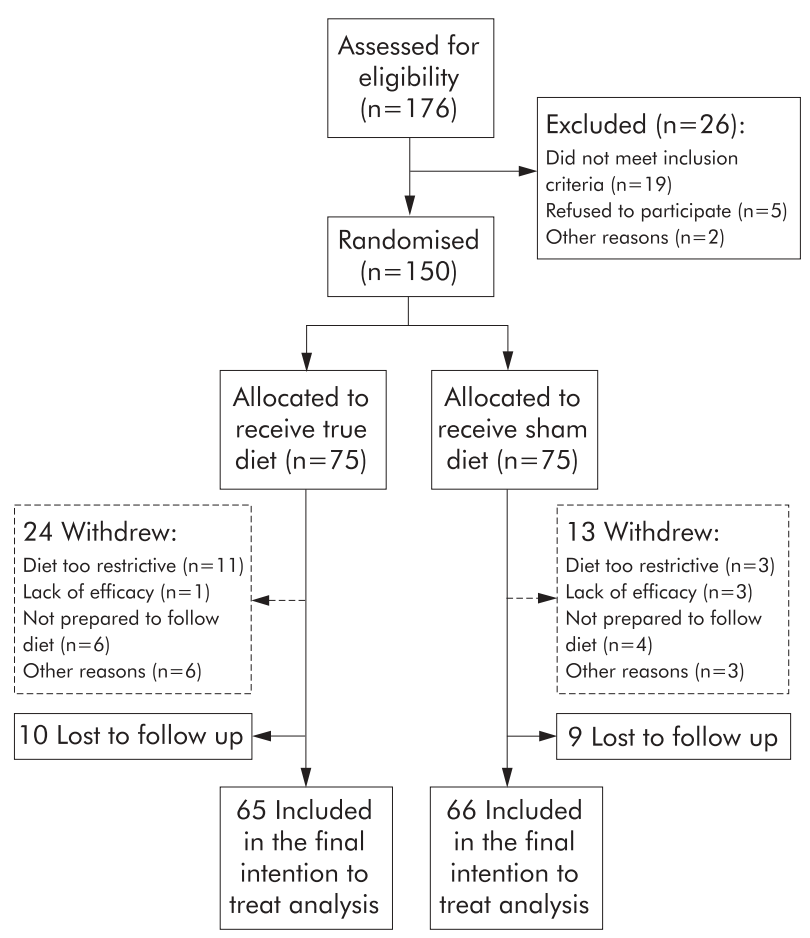

Figure 1 Study flow diagram. 


\begin{tabular}{|c|c|c|}
\hline Group & True diet $(n=75)$ & Sham diet $(n=75)$ \\
\hline Age (y) (range, SD) & $44(17-72 ; 12.9)$ & $44(19-74 ; 15.2)$ \\
\hline No of males (\%) & $7(9.3 \%)$ & $13(17.3 \%)$ \\
\hline No of foods to which sensitive & $6.65(3.66)$ & $6.63(4.1)$ \\
\hline Symptom duration (y) & $11.5(9.9)$ & $10.1(7.5)$ \\
\hline IBS symptom severity score & $331.9(70.8)$ & 309.0 (78.5) \\
\hline Non-colonic features score & $459.1(160.7)$ & $452.6(170.1)$ \\
\hline Quality of life score & $640.1(252.6)$ & $639.3(222.3)$ \\
\hline HAD anxiety score & $9.5(4.6)$ & $9.5(4.5)$ \\
\hline HAD depression score & $5.3(3.4)$ & $6.0(3.6)$ \\
\hline No of diarrhoea predominant patients (\%) & $37(52.1 \%)$ & $41(56.9 \%)$ \\
\hline No of constipation predominant patients (\%) & $19(26.8 \%)$ & $16(22.2 \%)$ \\
\hline No of alternating predominant patients (\%) & $15(21.1 \%)$ & $15(20.8 \%)$ \\
\hline
\end{tabular}

relationship between the change in symptoms from baseline and treatment group, patient characteristics (for example, IBS subtype, history of atopy, number of foods to which sensitive, and concomitant medication) and adherence to the diet. $^{30}$

\section{Sample size calculation}

It was estimated that approximately $40 \%$ of the placebo arm would report a significant improvement in symptoms. It was calculated that a sample size of 55 patients would be required in each group to detect, with $90 \%$ power, a difference of $30 \%$ points in the proportion reporting such an improvement (that is, $70 \%$ in the treatment arm) as statistically significant at the $5 \%$ level. Assuming a 20\% dropout rate, a minimum of 138 patients would need to be entered into the trial. Thus we aimed to recruit a total of 150 patients into the study.

\section{RESULTS}

Recruitment of patients and their flow through each stage of the study is illustrated in fig 1 , as recommended by the

\begin{tabular}{lcc}
$\begin{array}{l}\text { Table 2 } \\
\text { patients) }\end{array}$ & Frequency of foods excluded from the diet (\% of \\
\hline Food & & \\
\hline Barley & Treatment group & Sham group \\
Corn & 26.7 & 9.3 \\
Rice & 22.7 & 14.7 \\
Rye & 8 & 54.7 \\
Wheat & 8 & 25.3 \\
Milk & 49.3 & 8 \\
Beef & 84.3 & 1.3 \\
Chicken & 24 & 9.3 \\
Pork & 21.3 & 13.3 \\
Cabbage & 5.3 & 36 \\
Celery & 12 & 24 \\
Haricot bean & 5.3 & 21.3 \\
Pea & 17.3 & 14.7 \\
Potato & 38.6 & 1.3 \\
Soy bean & 9.3 & 61.3 \\
Tomato & 22.7 & 10.7 \\
Apple & 4 & 44 \\
Orange & 1.3 & 33 \\
Strawberry & 6.7 & 29.3 \\
Almond & 0 & 20 \\
Brazil nut & 28 & 12 \\
Cashew nut & 22.7 & 17.3 \\
Peanut & 49.3 & 8 \\
Walnut & 10.7 & 20 \\
Cocoa bean & 2.7 & 29.3 \\
Shellfish & 1.3 & 21.3 \\
Fish mix & 21.3 & 10.7 \\
Whole egg & 17.3 & 28 \\
Yeast & 57.3 & 26.7 \\
\hline & 86.7 & 0 \\
\hline & & \\
& &
\end{tabular}

CONSORT statement. ${ }^{31}$ In summary, between January 2001 and July 2002, 176 patients were eligible for the study, of which 26 (15\%) were excluded from participation, leaving 150 patients who were all found to be sensitive to at least one food. Seventy five of these were randomised to receive an elimination diet based on their true food sensitivity results and 75 patients to a sham diet. Data from 131 (87\%) patients who gave 12 week data were available for the intention to treat analysis: 65 and 66 patients from the true and sham groups, respectively.

\section{Patient characteristics}

The patients were typical of those with IBS in secondary care practice, the majority being women. Patients, on average, had experienced symptoms of IBS for over a decade and were found to be sensitive to approximately 6-7 foods (range 119). Baseline demographic and clinical characteristics of the two groups, including the use of concomitant medication, were found to be similar with the exception of the IBS symptom severity score which was slightly higher in the treatment group (table 1). Thirty per cent of patients were found to be atopic.

The frequency of foods excluded from the diet is shown in table 2. Adherence was lower in those on the true diet although no specific adverse events were recorded in either group. Twenty four patients withdrew from the study in the true diet group (mainly because of difficulty in following the diet) and 13 from the sham diet group (for a variety of reasons). However, 12 week data were obtained from 14 of those who withdrew in the true diet group and four in the sham diet group. There were no significant differences

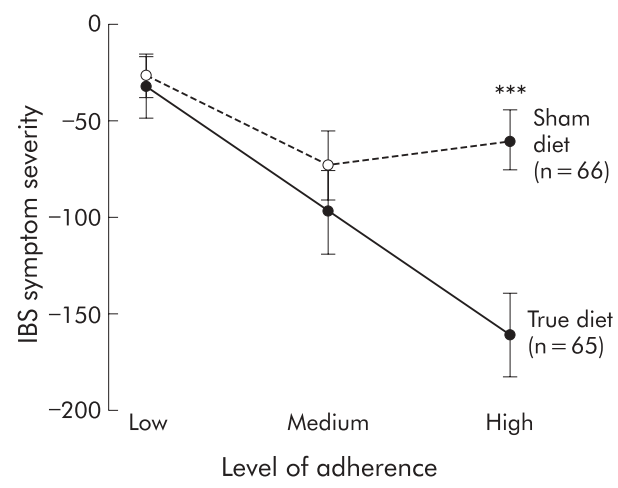

Figure 2 Mean change in symptom severity scores at 12 weeks according to degree of adherence. Difference between the groups with high adherence: 101 (95\% confidence interval 54, 147); ${ }^{* * *} \mathrm{p}<0.001$. 

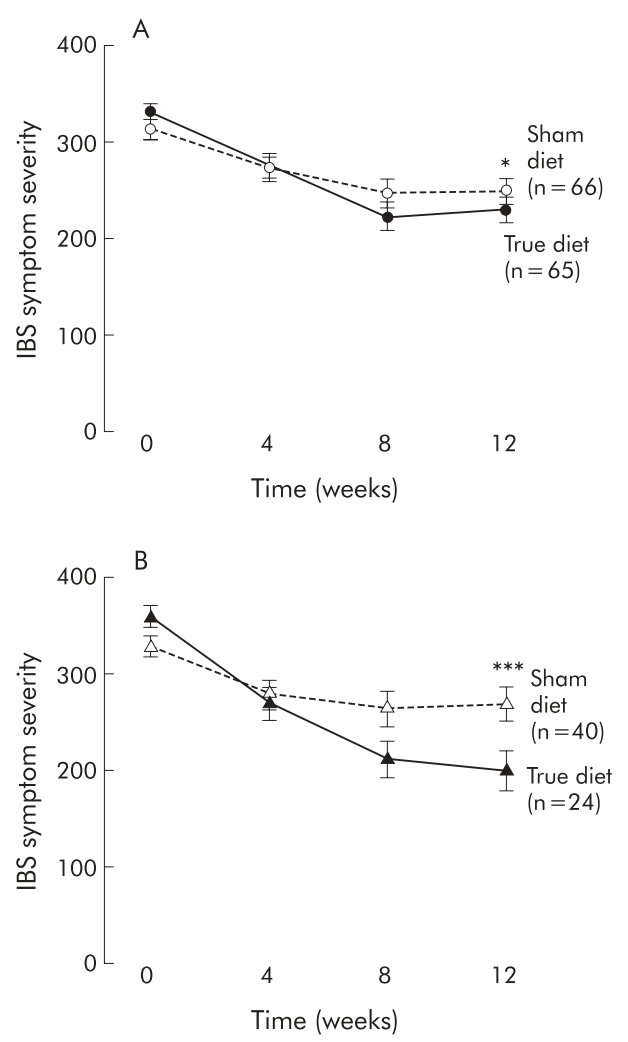

Figure 3 (A) Average symptom severity scores over time for the group as a whole. Difference in mean change from baseline at 12 weeks: true versus sham 39 (95\% confidence interval 5, 72); ${ }^{*} p=0.024$. (B) Average symptom severity scores over time for the full adherence group. Difference in mean change from baseline at 12 weeks: true versus sham $98(95 \%$ confidence interval 52,144$) ;{ }^{* * *} p<0.001$.

between baseline characteristics of the 19 who were lost to follow up and those for whom 12 week data were obtained.

\section{Primary outcomes}

IBS symptom severity

Patients in the true diet group experienced a $10 \%$ greater reduction in symptom severity than those allocated to the sham diet, with change in scores of 100 and 61.5, respectively (mean difference 39 (95\% confidence interval (CI) 5.2, 72.3); $\mathrm{p}=0.024$ ): a standardised effect size of 0.52 (see fig 3A). There were no differences in the response to the diet in terms of age, sex, IBS bowel habit subtype, or IBS duration. In addition, there was no difference in response to the diet between atopic and non-atopic patients. There was however a statistically significant interaction between treatment group and both adherence to the diet and number of foods to which patients were sensitive. For patients sensitive to the average number of foods who fully adhered to their allocated diet, a $26 \%$ difference in reduction in symptom severity score was observed in favour of the true diet (a difference in score of 98 (95\% CI 52, 144), p<0.001: a standardised effect size of 1.3). This benefit increased by a further 39 points ( $12 \%$ ) (95\% CI 7 , $70 ; p=0.016$ ) for each food to which they were sensitive over and above the average number. These results were not materially altered by carrying out an ANCOVA analysis (in which the final score is the dependent variable and the baseline score is included as a covariate) instead of modelling change in scores. ${ }^{30}$ The interaction between treatment group and adherence is demonstrated in fig 2 which shows a greater reduction in symptoms with full adherence in the true diet but not in the sham diet group. Figure $3 \mathrm{~A}$ and $3 \mathrm{~B}$ show the average change in symptom severity score over 12 weeks for the group as a whole and for those who fully adhered, respectively. This reveals that most improvements in symptoms are fully achieved within two months.

\section{Global impact score}

The reported global rating of change by treatment group is shown in table 3. The difference in mean ranking (70.9 $\mathrm{v}$ $60.3)$ was statistically significant $(p=0.048)$. When this was repeated including only patients who fully adhered to their diets (table 3 ), a greater percentage difference favouring the true diet was found $(\mathrm{p}=0.001)$. The NNT was 9 in the group as a whole and 2.5 in patients fully adherent to the diet.

\section{Secondary outcome measures}

As can be seen from fig $4 \mathrm{~A}$ and $4 \mathrm{~B}$, all data show changes favouring the true diet group and are consistent with the results for the primary outcomes. These trends were further strengthened after adjustment for adherence and number of food sensitivities but only reached statistical significance for non-colonic symptomatology $(p=0.05)$. There were no significant changes in medication use during the course of the trial.

\section{Reintroduction of eliminated foods}

Of the 131 patients who gave 12 week data, 93 (41 in the true and 52 in the sham diet groups) agreed to attempt reintroduction of foods they had been asked to eliminate and provided further follow up data on the primary outcomes measures. Of these, $62 \%$ reported full adherence and 37\% moderate adherence to the previous elimination diet. Mean IBS symptom severity score increased (that is, worsening of symptoms) by 83.3 in the true group and by 31 in the sham

Table 3 Global impact score at 12 weeks

\begin{tabular}{|c|c|c|c|}
\hline & \multicolumn{2}{|c|}{ Treatment group } & \\
\hline & $\begin{array}{l}\text { True diet } \\
\text { (n (\%)) }\end{array}$ & $\begin{array}{l}\text { Sham diet } \\
\text { (n }(\%))\end{array}$ & \\
\hline \multicolumn{4}{|l|}{ All patients } \\
\hline Significantly worse & $3(4.7)$ & $8(12.1)$ & \\
\hline No significant change & $44(67.2)$ & 47 (71.2) & \\
\hline Significantly improved & $18(28.1)$ & $11(16.7)$ & \\
\hline Total & 65 & 66 & NNT $=9$ \\
\hline \multicolumn{4}{|c|}{ Patients fully adhering to the diet } \\
\hline Significantly worse & $1(4.2)$ & $5(12.5)$ & \\
\hline No significant change & $10(41.7)$ & $29(72.5)$ & \\
\hline Significantly improved & $13(54.1)$ & $6(15)$ & \\
\hline Total & 24 & 40 & $\mathrm{NNT}=2.5$ \\
\hline
\end{tabular}



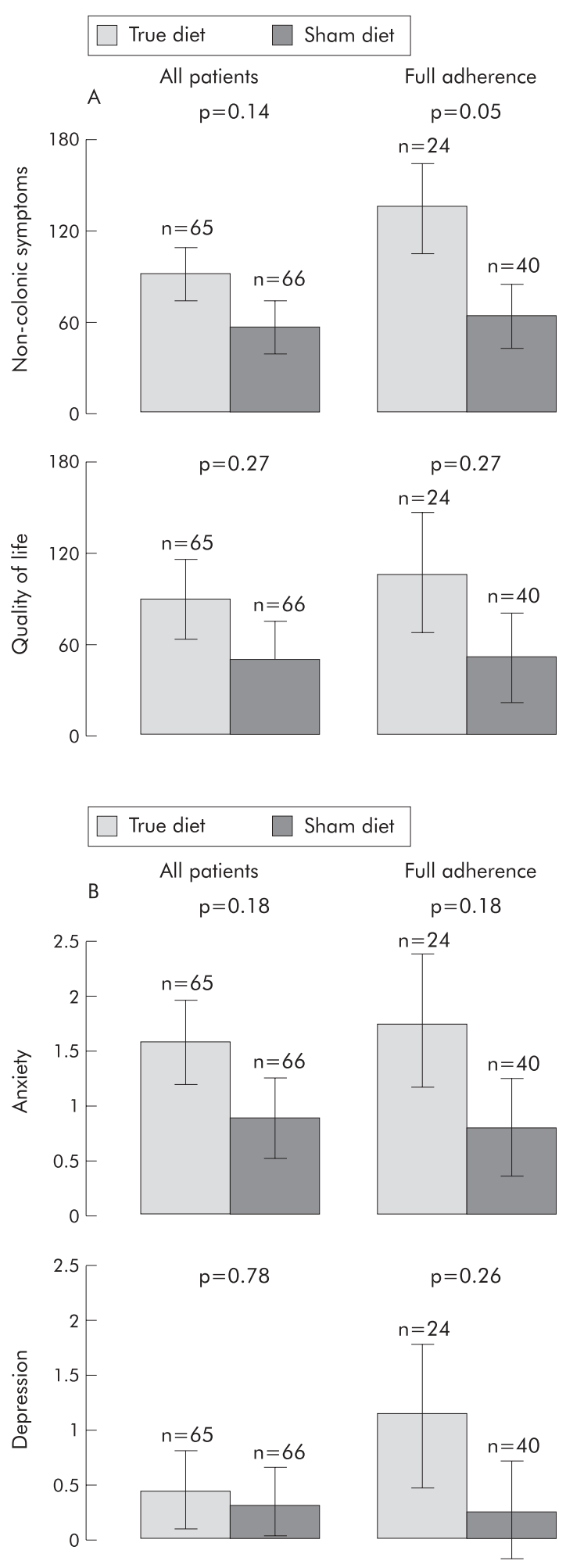

Figure 4 (A) Mean change in the secondary outcome measures of noncolonic symptoms and quality of life for the group as a whole and the full adherence group. (B) Mean change in the secondary outcome measures of anxiety and depression for the group as a whole and the full adherence group.

group, a statistically significant difference of 52 (24\%) (95\% CI 18, 86; $p=0.003)$. The change in global score following reintroduction of foods is shown in table 4 . This indicates a reversal of the pattern observed during the active treatment phase, with more patients in the true diet group showing
Table 4 Global rating following reintroduction of foods relative to the end of the elimination phase

\begin{tabular}{lll}
\hline & \multicolumn{2}{l}{ Treatment group } \\
\cline { 2 - 3 } & True diet group & \multicolumn{2}{l}{$\begin{array}{l}\text { Sham diet group } \\
\text { (n (\%)) }\end{array}$} & (n)) \\
\hline Significantly worse & $17(41.5)$ & $13(25)$ \\
No significant change & $23(56.1)$ & $35(67.3)$ \\
Significantly improved & $1(2.4)$ & $4(7.7)$ \\
Total & $41(100)$ & $52(100)$ \\
\hline & & \\
\hline
\end{tabular}

worsening of health compared with the sham diet group $(\mathrm{p}=0.047)$.

\section{DISCUSSION}

A clinically significant improvement in IBS symptomatology was observed in patients eliminating foods to which they were found to exhibit sensitivity, as identified by an ELISA test for the presence of IgG antibodies to these foods. The number needed to treat of 9 for the group as a whole and 2.5 for patients closely adhering to the diet are both considerably better than the value of 17 achieved after three months of treatment with tegaserod, ${ }^{32}$ a drug that has been recently licensed in the USA for use in IBS. IBS symptom severity and global rating scores were chosen as primary outcome measures in this study as they represented the most direct measure of clinical improvement in this condition based on patient self assessment. Rather than using the traditional method of classifying global improvement as any value exceeding adequate relief of symptoms, we used a much stricter definition requiring patients to report symptoms as being either "better" or "excellent" compared with pretreatment levels. Despite this, the diet still achieved a significant improvement. However, as might be expected, the placebo response using this end point was somewhat lower than that usually reported in IBS treatment trials which have used less demanding criteria. The observation that patients on the sham diet also improved, although to a lesser extent, emphasises the importance of conducting double blind randomised controlled trials of such non-drug interventions in order to avoid overestimating their potential.

Most patients with IBS have attempted at least some form of dietary modification, which in some cases can be very extreme. Conflicting results have been reported using exclusion $\operatorname{diets}^{453-36}$ and this approach also suffers from the limitation that it has to be empirical. Thus potentially offending foods can only be identified after their elimination and subsequent reintroduction. This time consuming process would be much reduced if the offending foods could be identified beforehand. Attempts to do this using IgE antibodies have been disappointing ${ }^{8-10}$ but the results of this study suggest that measuring IgG antibodies may be much more rewarding. The response to the IgG based diet in our trial did not correlate with atopic status, the prevalence of which was found to be no greater than that occurring in the general population. ${ }^{37}$

The observation that adherence to the diet is critical in determining a good outcome in the "true" diet group but not the "sham" group is indicative of the fact that the diet is an "active treatment" which if not adhered to, does not seem to have an effect. This notion is further supported by the observation that a significantly greater deterioration was observed in subjects in the true diet group compared with those in the sham group when they reintroduced eliminated foods at the end of the diet phase of the trial. Furthermore, the improvement of 98 in the symptom severity score in those fully adherent in the true diet group is well above the value of 
50, which is regarded as being of clinical significance both in validation studies ${ }^{24}$ and clinical practice. ${ }^{26-28}$ It was interesting to note that patients exhibiting a greater number of sensitivities, as determined by the IgG test, experienced a greater symptom reduction if they adhered to the true but not the sham diet.

There is currently considerable interest in the concept that at least in some patients, IBS may have an inflammatory component. ${ }^{38-42}$ Most of the work in this area has centred on post dysenteric IBS, with gut pathogens being viewed as the initiators of this process which can be identified by subtle changes on histology. ${ }^{38}$ However, if, as indicated in this study, IgG antibodies to food are important in the pathogenesis of IBS in some patients, they too may be of relevance. Not all patients exhibiting histological features consistent with post dysenteric IBS give a history of a previous dysenteric illness. This is usually assumed to be due to the fact that this has been forgotten by the patient but our results may suggest an alternative mechanism for immune activation and inflammation without the need for prior infection.

It is now well recognised that up to $70 \%$ of patients with IBS have evidence of hypersensitivity of the rectum, ${ }^{43}$ which probably extends to involve most of the gut in many individuals. ${ }^{44}$ It is possible that this hypersensitivity renders patients more reactive to a low grade inflammatory process which would not necessarily cause symptoms in a normal individual. This would explain why excluding foods to which patients have IgG antibodies might be particularly beneficial in IBS despite the fact that these antibodies may also be present in the general population. Indeed, if this mechanism is particularly important in IBS, it might be anticipated that IgG food antibodies would be relatively common in this condition, as was the case in our study.

Many patients with IBS would prefer a dietary solution to their problem rather than having to take medication, and the economic benefits of this approach to health services are obvious. It is well known that patients expend large sums of money on a variety of unsubstantiated tests in a vain attempt to identify dietary intolerances. The results of this study suggest that assay of IgG antibodies to food may have a role in helping patients identify candidate foods for elimination and is an approach that is worthy of further biomedical and clinical research.

\section{Authors' affiliations}

W Atkinson, N Shaath, P J Whorwell, Department of Medicine, University Hospital of South Manchester, Manchester, UK T A Sheldon, Department of Health Sciences, University of York, York, UK

\section{REFERENCES}

1 Drossman DA, Camilleri M, Mayer EA, et al. American Gastroenterological Association Technical Review on Irritable Bowel Syndrome. Gastroenterology 2002; 123:2108-31.

2 Lea R, Whorwell PJ. Quality of life in irritable bowel syndrome. Pharmacoeconomics 2001;19:643-53.

3 Talley NJ, Spiller R. Irritable bowel syndrome: a little understood organic bowel disease? Lancet 2002;360:555-64.

4 Jones VA, McLaughlan P, Shorthouse M, et al. Food intolerance: a major factor in pathogenesis of irritable bowel syndrome. Lancet 1982;2:1115-17.

5 Nanda R, James R, Smith $\mathrm{H}$, et al. Food intolerance and the irritable bowel syndrome. Gut 1989;30:1099-104.

6 Zwetchkenbaum J, Burakoff, R. The irritable bowel syndrome and food hypersensitivity. Ann Allerg 1988;61:47-9.

7 Zar S, Kumar D, Benson M. J. Review article: food hypersensitivity and irritable bowel syndrome, Aliment Pharm Ther 2001;15:439-43.

8 Petitpierre M, Gumowski P, Girard JP. Irritable bowel syndrome and hypersensitivity to food. Ann Allergy 1985;54:538-40.

9 Barau E, Dupont C. Modifications of intestinal permeability during food provocation procedures in pediatric irritable bowel syndrome. J Pediatr Gastroenterol Nutr 1990;11:72-7.

10 Roussos A, Koursarakos P, Patsopoulos D, et al. Increased prevalence of irritable bowel syndrome in patients with bronchial asthma. Respir Med 2003;97:75-9
11 Crowe SE, Perdue MH. Gastrointestinal food hypersensitivity: basic mechanisms of pathophysiology. Gastroenterol 1992;103:1075-95.

12 el Rafei A, Peters SM, Harris N, et al. Diagnostic value of lgG4 measurements in patients with food allergy. Ann Allergy 1989;62:94-9.

13 Host A, Husby S, Gjesing B, et al. Prospective estimation of lgG, lgG subclass and IgE antibodies to dietary proteins in infants with cow's milk allergy. Levels of antibodies to whole milk protein, BLG and ovalbumin in relation to repeated milk challenge and clinical course of cow's milk allergy. Allergy 1992;47:218-29.

14 Awazuhara H, Kawai H, Maruchi N. Major allergens in soybean and clinical significance of $\lg G 4$ antibodies investigated by $\lg E$ and $\lg G 4$ immunoblotting with sera from soybean-sensitive patients. Clin Exp Allergy 1997;27:325-32.

15 Barnes RMR, Johnson PM, Harvey MM, et al. Human serum antibodies reactive with dietary proteins: IgG subclass distribution. Int Arch Allergy Appl Immunol 1988;87:184-8.

16 Lessof MH, Kemeny DM, Price JF. IgG antibodies to food in health and disease. Allergy Proc 1991;12:305-7.

17 Husby S, Mestecky J, Moldoveanu Z, et al. Oral tolerance in humans. T cell but not $B$ cell tolerance after antigen feeding. J Immunol 1994;152:4663-70.

18 Haddad ZH, Vetter M, Friedmann J, et al. Detection and kinetics of antigenspecific lgE and lgG immune complexes in food allergy. Ann Allergy 1983;51:255.

19 Husby S, Oxelius VA, Teisner B, et al. Humoral immunity to dietary antigens in healthy adults. Occurrence, isotype and lgG subclass distribution of serum antibodies to protein antigens. Int Arch Allergy Appl Immunol 1985;77:416-22

20 Kruszewski J, Raczka A, Klos $M$, et al. High serum levels of allergen specific lgG-4 (aslgG-4) for common food allergens in healthy blood donors. Arch Immunol Ther Exp 1994:42:259-61.

21 Finn R, Smith MA, Youngs GR, et al. Immunological hypersensitivity to environmental antigens in the irritable bowel syndrome. $\mathrm{Br} J$ Clin Pract 1987;41:1041-3.

22 Drossman DA, Corazziari E, Talley NJ, et al. Rome II: a multinational consensus document on functional gastrointestinal disorders. Gut 1999;45:1-81

23 Foster AP, Knowles TG, Hotston Moore A, et al. Serum lgE and lgG responses to food antigens in normal and atopic dogs, and dogs with gastrointestinal disease. Vet Immunol Immunopathol 2003:92:113-24.

24 Francis CY, Morris J, Whorwell PJ. The irritable bowel scoring system: A simple method of monitoring IBS and its progress. Aliment Pharmacol Therap 1997; 11:395-402.

25 Whorwell PJ, McCallum H, Creed FH, et al. Non-colonic features of irritable bowel syndrome. Gut 1986;27:452-6.

26 Houghton LA, Heyman DJ, Whorwell PJ. Symptomatology, quality of life and economic features of irritable bowel syndrome-the effect of hypnotherapy. Aliment Pharmacol Ther 1996;10:91-5.

27 Gonsalkorale WM, Toner BB, Whorwell PJ. Cognitive change in patients undergoing hypnotherapy for irritable bowel syndrome. J Psychosom Res 2004;56:271-8.

28 Gonsalkorale WM, Houghton LA, Whorwell PJ. Hypnotherapy in irritable bowel syndrome: a large scale audit of a clinical service with examination of factors influencing responsiveness. Am J Gastroenterol 2002;97:954-61.

29 Zigmond AS, Snaith RP. The hospital anxiety and depression scale. Acta Psychiatr Scand 1983;67:361-70.

30 Everitt BS, Pickles A. Statistical aspects of the design and analysis of clinical trials. London: Imperial College Press Publishers, 2003:108-42.

31 Altman DG, Schulz KF, Moher D, et al. The revised CONSORT statement for reporting randomized trials: explanation and elaboration. Ann Intern Med 2001; 134:663-94.

32 Novick J, Miner P, Krause R, et al. A randomised, double blind, placebo controlled trial of tegaserod in female patients suffering from irritable bowe syndrome with constipation. Aliment Pharmacol Ther 2002;16:1877-88.

33 Niec AM, Frankum B, Talley NJ. Are adverse reactions to food linked to irritable bowel syndrome? Am J Gastroenterol 1998;93:2184-90.

34 Burden S. Dietary treatment of irritable bowel syndrome: current evidence and guidelines for future practice. J Hum Nutr Diet 2001;14:231-41.

35 Bentley SJ, Pearson DJ, Rix KJB. Food hypersensitivity in irritable bowel syndrome. Lancet 1983;2:295-7.

36 McKee AM, Prior A, Whorwell PJ. Exclusion diets in irritable bowel syndrome: Are they worthwhile? J Clin Gastroenterol 1987;9:526-8.

37 Durham SR, Church MK. Principles of allergy diagnosis. In: Holgate ST, Church MK, Lichtenstein LM, eds. Allergy, 2nd edn. London: Mosby, 2001:3-16.

38 Spiller RC, Jenkins D, Thornley JP, et al. Increased rectal mucosal enteroendocrine cells, T lymphocytes, and increased gut permeability following acute Campylobacter enteritis and in post-dysenteric irritable bowel syndrome. Gut 2000;47:804-11

39 Gonsalkorale WM, Perrey C, Pravica V, et al. Interleukin 10 genotypes in irritable bowel syndrome: evidence for an inflammatory component? Gut 2003:52:91-3.

40 Collins SM, Piche T, Rampal P. The putative role of inflammation in the irritable bowel syndrome. Gut 2001;49:743-5.

41 Collins SM. A case for an immunological basis for irritable bowel syndrome. Gastroenterology 2002;122:2078-80.

42 Chadwick VS, Chen W, Shu D, et al. Activation of the mucosal immune system in irritable bowel syndrome. Gastroenterology 2002;122:1778-83.

43 Mertz H. Review article: visceral hypersensitivity. Aliment Pharmacol Ther 2003; 17:623-33.

44 Francis CY, Houghton LA, Whorwell PJ. Enhanced sensitivity of the whole gut in patients with irritable bowel syndrome. Gastroenterology 1995; 108:601 (abstract) 


\section{PostScript}

\section{LETTERS}

\section{ITPA genotyping test does not improve detection of Crohn's disease patients at risk of azathioprine/6-mercaptopurine induced myelosuppression}

The thiopurine drugs azathioprine (AZA) and 6-mercaptopurine (6-MP) are effective for the treatment of inflammatory bowel disease (IBD) and their prescription is increasing. Haematotoxicity, which can lead to potentially life threatening bone marrow suppression, represents the most serious side effect of thiopurine therapy. It has been attributed to the accumulation of active cytotoxic metabolites of AZA/6-MP, collectively called 6-thioguanine nucleotides, resulting from a deficiency in thiopurine catabolism specifically catalysed by the thiopurine $S$-methyltransferase (TPMT) enzyme. Genotyping tests are now available to identify deficient and intermediate methylators who are, respectively, homozygous and heterozygous for non-functional alleles of the TPMT gene. As pointed out by Lennard in the leading article (Gut 2002;51:143-6), it is clear that myelosuppression may be caused by other factors in addition to variable TPMT.

Since the identification of the molecular basis of inosine triphosphate pyrophosphatase (ITPAse) deficiency, ${ }^{1}$ a clinically benign condition characterised by abnormal accumulation of inosine triphosphate in erythrocytes, the possibility of a correlation between thiopurine toxicity and ITPAse deficiency has been raised. Complete ITPase deficiency was found to be associated with a homozygous missense 94C $>$ A mutation that encodes a $\mathrm{Pro}^{32} \mathrm{Thr}$ exchange, whereas an intronic IVS2+21 A $>C$ polymorphism was shown to have a less severe effect, homozygotes retaining $60 \%$ ITPAse activity. It was then postulated that in ITPAse deficient patients treated with thiopurine drugs, a 6-thio-ITP metabolite could accumulate resulting in toxicity. ${ }^{1} \mathrm{~A}$ recent study in 62 patients with inflammatory bowel disease reported a significant association between the ITPA $94 \mathrm{C}>\mathrm{A}$ polymorphism and AZA related adverse effects, specifically flu-like symptoms, rash, and pancreatitis. $^{2}$ No correlation was observed with occurrence of neutropenia but only 11 patients were studied. We previously reported TPMT genotype analysis in 41 Crohn's disease

Table 1 Distribution of ITPA genotypes in 41 Crohn's disease (CD) patients and 100 healthy Caucasians

\begin{tabular}{lcl}
\hline ITPA genotype & CD patients $(n=41)$ & Control populationt $(\mathbf{n}=100)$ \\
\hline$W_{t} / W_{t}$ & $26(0.63)^{*}$ & $64(0.64)$ \\
$W_{t} / 94 C>A$ & $6(0.15)$ & $10(0.10)$ \\
$W_{t} / I V S 2+21 A>C$ & $7(0.17)$ & $24(0.24)$ \\
$94 C>A / 94 C>A$ & $0(0.00)$ & $0(0.00)$ \\
IVS2+21A $>C / I V S 2+21 A>C$ & $1(0.02)$ & $0(0.00)$ \\
$94 C>A / I V S 2+21 A>C$ & $1(0.02)$ & $2(0.02)$ \\
\hline
\end{tabular}

*Values in parentheses represent genotype frequencies.

†The control population comprised 100 healthy Caucasians who were genotyped in a previous study.
(CD) patients who had experienced leucopenia during AZA/6-MP therapy. ${ }^{3}$ Even though this study confirmed the efficiency of TPMT genotyping in identifying patients at risk of developing myelosuppression, it also highlighted its limitations, as only $27 \%$ of patients carried mutant alleles of the TPMT gene that were associated with enzyme deficiency. This prompted us to investigate the occurrence of ITPA mutations in this series of patients in order to evaluate whether genotyping of the ITPAse gene could improve the detection rate of patients at risk of thiopurine myelotoxicity.

Our population comprising $4 \mathrm{l}$ patients with $C D$ has been described in detail previously. ${ }^{3}$ Briefly, all patients had either leucopenia (white blood cell count $<3000$ / $\mathrm{mm}^{3} ; \mathrm{n}=24$ ) or thrombocytopenia (platelets $\left.<100000 / \mathrm{mm}^{3} ; \mathrm{n}=30\right)$, or both $(\mathrm{n}=14)$, leading either to discontinuation of treatment or reduction of dose by $50 \%$ or more during AZA $(\mathrm{n}=33)$ or 6 -MP $(\mathrm{n}=8)$ treatment. Patients were genotyped for the ITPA $94 \mathrm{C}>\mathrm{A}$ and IVS2+21 A $>C$ mutations according to a previously described procedure based on endonuclease digestion of polymerase chain reaction products. ${ }^{1}$ Distribution of the 41 patients according to their ITPA genotype is presented in table 1 and compared with that of a previously published control population of 100 healthy Caucasians. ${ }^{1}$ Allele frequencies in the $\mathrm{CD}$ population were 0.085 for the $94 \mathrm{C}>\mathrm{A}$ mutation and 0.12 for the IVS $2+21 \mathrm{~A}>\mathrm{C}$ mutation, similar to frequencies observed in the control population $(0.06$ and 0.13 , respectively). There was no significant difference in the genotypes distribution between the two populations, which confirmed the lack of association between ITPAse deficiency and myelosuppression during thiopurine therapy. Due to the retrospective nature of the study, no correlation with other side effects could be investigated.

In conclusion, application of ITPA genotyping tests does not seem to improve the identification of patients at risk of myelosuppression with AZA/6-MP therapy. Although we believe that conventional TPMT genotyping tests should still be applied before the initiation of thiopurine treatment, further work is needed on the role of other candidate genes that may be involved in thiopurine haematotoxicity.

\section{Acknowledgements}

We thank $\mathrm{N}$ Ferrari and A Vincent for their assistance in performing the study and the members of the GETAID for recruiting patients in the study.
D Allorge, R Hamdan, F Broly EA2679, Faculté de Médecine/Pôle Recherche, Place de Verdun, Lille, France

C Libersa

Centre d'Investigations Cliniques, Hôpital Cardiologique, CH et U Lille, Lille, France

J-F Colombel

Service d'Hépato-Gastroentérologie, Hôpital Huriez, $\mathrm{CH}$ et $\mathrm{U}$ Lille, Lille, France

Groupe d'Etudes Thérapeutiques des Affections Inflammatoires Digestives (GETAID) Service d'Hépato-Gastroentérologie, Hôpital Saint-Louis, Paris, France

Correspondence to: Professor J-F Colombel, Service d'Hépato-Gastroentérologie, Hôpital Huriez, CH et U Lille, 59037 Lille, France; ifcolombel@chru-lille.fr

doi: $10.1136 /$ gut.2004.055947

Competing Interests: None declared.

\section{References}

1 Sumi S, Marinaki AM, Arenas M, et al. Genetic basis of inosine triphosphate pyrophosphohydrolase deficiency. Hum Genet 2002;111:360-7.

2 Marinaki AM, Ansari A, Duley JA, et al. Adverse drug reactions to azathioprine therapy are associated with polymorphism in the gene encoding inosine triphosphate pyrophosphatase (ITPase). Pharmacogenetics 2004; 14:181-7.

3 Colombel JF, Ferrari N, Debuysere $\mathrm{H}$, et al. Genotypic analysis of thiopurine Smethyltransferase in patients with Crohn's disease and severe myelosuppression during azathioprine therapy. Gastroenterology 2000;118:1025-30.

\section{Small bowel malignancy at diagnosis of coeliac disease}

We were very interested in the paper by Rampertab et al (Gut 2003;52:121-14) and the correspondence by Hawdle et al (Gut 2004;53:470). Their data are quite similar to ours, from the Italian Registry of Complications of Coeliac Disease.

We collected information on 1968 patients over 18 years of age (mean age at diagnosis: 36.7 years; female/male ratio 3:1), diagnosed with coeliac diseases between January 1982 and December 2002 at 20 Italian clinical centres specialised in gastrointestinal disease. The diagnosis was made according to revised ESPGHAN criteria. ${ }^{1}$ We found five $(0.25 \%)$ patients with a small bowel malignancy at the time of diagnosis of coeliac disease. Age range was 49-69 years (mean 59 years) with a predominance of females (4:1). Survival rate was very poor as three patients died within 36 months of diagnosis.

These results indicate that there is an increased risk of developing small bowel malignancy in patients with coeliac disease. This correlation was confirmed by the female/ male ratio. In fact, while small bowel neoplasms are usually more frequent in males, in our population four of five cases were female. Moreover, mean age at diagnosis of these cases was higher than that of patients overall, emphasising that the risk of a neoplasm increases with longstanding coeliac disease. 
In conclusion, early diagnosis of coeliac disease should be made to prevent small bowel neoplasms from developing, and screening for this cancer should be carried out at diagnosis of coeliac disease, especially in patients diagnosed during adulthood.

M Silano, M De Vincenzi

Division of Human Nutrition and Health, Istituto Superiore di Sanità, Rome, Italy

Correspondence to: Dr M Silano, Division of Human Nutrition and Health, Istituto Superiore di Sanita', Viale Regina Elena, 299, 00161 Roma, Italy; marco.silano@iss.it

Competing Interests: None declared.

\section{Reference}

1 Walker-Smith J, Guandalini S, Schmitz J, et al. Revised criteria for diagnosis of coeliac disease. Report of Working Group of European Society of Paediatric Gastroenterology and Nutrition.

J Pediatr Gastroenterol Nutr 1990;65:909-11.

\section{Hypergastrinaemia in patients infected with Helicobacter pylori treated with proton pump inhibitors}

We read with interest the commentary by McColl on Helicobacter pylori infection and long term proton pump inhibitor (PPI) therapy (Gut 2004;53:5-7).

It is remarkable that he did not mention gastrin although hypergastrinaemia is a result of reduced gastric acidity ${ }^{1}$ as well as Helicobacter pylori infection, ${ }^{2}$ and that patients with $H$ pylori infection treated with PPI have additive hypergastrinaemia. ${ }^{3}$ Hypergastrinaemia predisposes to gastric carcinoids in animals $^{45}$ and humans ${ }^{67}$ as well as to malignant ECL cell derived tumours (gastric carcinomas) in animals ${ }^{8}$ and humans. ${ }^{9} 10$

Interestingly, the carcinogenic effect of $H$ pylori infection may be completely explained by its hypergastrinaemic effect, ${ }^{11}$ a work where McColl was one of the authors. Furthermore, the increased gastric cancer frequency in moderate hypergastrinaemic INS-GAS mice concomitantly infected by $H$ pylori infection ${ }^{12}$ may also be caused by increased hypergastrinaemia in infected mice. $^{13}$

To conclude, it is odd that gastrin was not taken into consideration when discussing the risk of gastric cancer following treatment with PPI in patients infected with $H$ pylori. Animal as well as human studies linking gastrin to gastric cancer give support for a strategy where $H$ pylori is eradicated in patients on long term PPI treatment.

\section{H L Waldum}

Correspondence to: Professor H L Waldum, Norwegian University of Science and Technology, Department of Clinical and Molecular Medicine, Trondheim University, Trondheim N-7006, Norway; helge.waldum@medisin.ntnu.no

Competing Interests: None declared.

\section{References}

1 Korman MG, Strickland RG, Hansky J. Serum gastrin in chronic gastritis. BMJ 1971;2:16-18.

2 Moss SF, Calam J. Acid secretion and sensitivity to gastrin in patients with duodenal lulcer: effect of eradication of Helicobacter pylori. Gut 1993;34:888-92
3 Schenk BE, Kuipers EJ, Klinkenberg-Knol EC, et al. Hypergastrinaemia during long-term omeprazole therapy: influences of vagal nerve function, gastric emptying and Helicobacter pylori infection. Aliment Pharmacol Ther

1998; 12:605-12.

4 Havu N. Enterochromaffin-like cell carcinoids of gastric mucosa in rats after life-long inhibition of gastric acid secretion. Digestion

1986;35(suppl):42-55.

5 Hirayama F, Takagi S, Iwao E, et al. Development of poorly differentiated adenocarcinoma and carcinoid due to long-term Helicobacter pylori colonization in Mongolian gerbils. J Gastroenterol 1999;24:450-4.

6 Sjöblom S-M, Sipponen P, Karonen S-L, et al. Argyrophil cell hyperplasia and carcinoid tumours in oxyntic mucosa of the stomach. Dependence on duration of pernicious anaemia. Eur J Gastroenterol Hepatol 1991;31:153-7.

7 Cadiot G, Vissuzaine C, Potet F, et al. Fundic argyrophic carcinoid tumor in a patient with sporadic-type Zollinger-Ellison syndrome. Dig Dis Sci 1995;40:1275-8.

8 Waldum HL, Rørvik H, Falkmer S, et al. Neuroendocrine (ECL-cell) differentiation of spontaneous gastric carcinomas of cotton rats (Sigmodon hispidus). Lab Anim Sci 1999;49:241-7.

9 Qvigstad G, Qvigstad T, Westre B, et al. Neuroendocrine differentiation in gastric adenocarcinomas associated with severe hypergastrinemia and/or pernicious anemia. APMIS 2002;110:132-9.

10 Qvigstad G, Falkmer S, Westre B, et al. Clinical and histopathological tumour progression in $\mathrm{ECL}$ cell carcinoids ("ECLomas"). APMIS 1999;107:1085-93.

11 Hansen S, Vollset SE, Ardill JES, et al. Hypergastrinemia is a strong predictor of distal gastric adenocarcinoma among Helicobacter pylori infected persons. Gastroenterology 1997; 1 12:A575.

12 Wang TC, Dangler CA, Chen D, et al. Synergistic interaction between hypergastrinemia and Helicobacter infection in a mouse model of gastrin carcinoma. Gastroenterology 2000; 1 18:36-47.

13 Waldum HL, Brenna E, Martinsen TC. Safety of proton pump inhibitors. Aliment Pharmacol Ther 2000; 14:1537-8.

\section{Terminal ileal biopsies should not} be used to document extent of colonoscopic examination

We commend the British Society of Gastroenterology and the authors for the excellent publication of guidelines for the management of inflammatory bowel disease in adults (Gut 2004;53(suppl V):vi-16). However, we feel that their recommendation for routine terminal ileal biopsying is inappropriate. Although it is important to biopsy the terminal ileum if there is macroscopic evidence of an abnormality, their statement that "a terminal ileal biopsy performed at colonoscopy documents the extent of examination" is not recommended practice, due to the potential risk of variant Creutzfeld-Jacob disease transmission from prion proteins which are prevalent in the lymphoid tissue of Peyer's patches in the ileum. Although the use of disposable forceps may reduce the risk of transmission, there could still be contamination of the intubation channel of the colonoscope and prion protein is resistant to the standard endoscopic cleaning process. ${ }^{1}$ If the extent of examination needs to be documented, then a photograph of the ileocaecal valve or ileal mucosa is preferable.

It is worth emphasising that prion protein may be present in any part of the gastrointestinal tract $^{2}$ and random biopsy of gastrointestinal mucosa for reasons other than confirming an endoscopic abnormality or excluding microscopic colitis is not accepta- ble. Similarly, for surveillance colonoscopy where multiple biopsy is recommended, the risk benefit ratio of this policy must be supported by the clinical indications.

M D Rutter

University Hospital of North Tees, Stockton-on-Tees, Cleveland, UK

M G Bramble

James Cook University Hospital, Middlesbrough, Cleveland, UK

Correspondence to: Dr M D Rutter, University Hospital of North Tees, Stockton-on-Tees, Cleveland, TS19 8PE, UK; matt.rutter@nth.nhs.uk

Competing Interests: None declared.

\section{References}

1 Bramble MG, Ironside JW. Creutzfeld-Jakob disease: implications for gastroenterology. Gut 2002;50:888-90.

2 Herzog C, Sales N, Etchegaray N, et al. Tissue distribution of bovine spongiform encephalopathy agent in primates after intravenous or oral infection. Lancet 2004;363:422-8.

\section{$\lg G$ food antibodies should be studied in similarly treated groups}

The recent paper by Atkinson and colleagues (Gut 2004;53:1459-1464) regarding IgG food antibodies and irritable bowel syndrome (IBS) fails to compare like with like. Regardless of the IgG results, the treatment group excluded significantly different foods to the control group, particularly those foods which appear to exacerbate symptoms of IBS. Of particular concern is the "yeast exclusion" diet. A low yeast diet is not a recognised diet in standard textbooks of dietetics and nutrition. However, alternative practitioners offering such a "yeast exclusion diet" sometimes recommend exclusion of a wide range of foods, such as: bakery products, alcoholic beverages, many other beverages including commercial fruit juices, cereals, condiments, dairy produce, fungi, meat products (hamburgers, sausages, and cooked meats made with bread or breadcrumbs), yeast extracts (Bisto, Marmite, Oxo, Bovril, Vegemite, gravy browning, and all similar extracts), all B vitamin preparations, and sometimes, most worryingly, "sugar foods" (sugar, sucrose, fructose, maltose, lactose, glycogen, glucose milk, sweets, chocolate, sweet biscuits, cakes, candies, cookies, puddings, desserts, canned food, packaged food, hamburgers, honey, mannitol, sorbitol, galactose, monosaccharides, polysaccharides, date sugar, turbinado sugar, molasses, maple syrup, most bottled juices, all soft drinks, tonic water, milkshakes, raisins, dried apricots, dates, prunes, dried figs, and other dried fruit).

Therefore, regardless of IgG antibody status, the dietary restrictions in one group are not controlled for by the other group, and hence the conclusion may not be valid.

It would also be helpful to know if any of the patients with IgG antibodies to a particular antigen also had IgE antibodies to the same antigen.

W A C Sewell

Correspondence to: Dr W A C Sewell, Path Links Immunology, Scunthorpe General Hospital, Scunthorpe DN15 7BH, UK; carrock.sewell@nlg.nhs.uk

Competing Interests: None declared. 


\section{IgG antibodies to foods in IBS}

We read with interest the article by Atkinson et al (Gut 2004;53:1459-64). The authors describe an important advance in our understanding of the putative role of inflammation in irritable bowel syndrome (IBS). However, we wonder whether their conclusion that assay of IgG antibodies may have a role in identifying candidate foods for elimination to treat patients with IBS may be a step too far. The four foods to which the patients most commonly formed antibodies and hence the four foods most commonly eliminated from the "true diet" were yeast $(86.7 \%)$, milk $(84.3 \%)$, whole egg $(58.3 \%)$, and wheat (49.3\%). The "sham diet" involved eliminating foods to which the patients had not formed antibodies and, therefore, in the sham group the exclusion rates for yeast, milk, whole egg, and wheat were very low $(0 \%, 1.3 \%, 26.7 \%$, and $8 \%$ respectively). It is therefore difficult to assess whether a diet excluding these foods would have led to symptomatic improvement in all patients, regardless of their antibody status.

Furthermore, the foods to which the study group commonly formed antibodies were similar to those already identified as leading to symptomatic benefit in patients with IBS when excluded from their diet. In a review cited by Atkinson and colleagues, ${ }^{1}$ it was noted that in eight trials of exclusion diets in IBS, seven identified dairy products and five identified wheat as worsening symptoms. It is not clear whether the difference in improvement in symptoms seen in the current study between true and sham groups can be explained simply by the omission of these foods. This could in practice eliminate the need for antibody testing.

J E D Mawdsley, P Irving, R Makins Barts and the London School of Medicine and Dentistry, London, UK

Correspondence to: Dr J E D Mawdsley, St Bartholomew's and Royal London Hospital, Turner St, London El 2AD, UK; joelmawdsley@yahoo.com

Competing Interests: None declared.

\section{Reference}

1 Burden S. Dietary treatment of irritable bowel syndrome: current evidence and guidelines for future practice. J Hum Nutr Diet 2001; 14:231-41.

\section{Influence of dietary factors on the clinical course of inflammatory bowel disease}

Jowett et al reported in their elegant study on the role of diet in maintaining remission in patients with ulcerative colitis (Gut 2004;53:1479-84). Surely the effect of diet has an essential, but often forgotten, role in altering the course of disease in all types of inflammatory bowel diseases. This role does not necessarily act by maintaining patients in remission clinically, but perhaps more importantly by minimising the activities of the disease and rendering it quiescent.

We have recently reported a case of active stricturing Crohn's disease in an adult female patient with high stoma output. ${ }^{1}$ She was treated solely with casein base formula (Modulen IBD-Nestle, Vevey, Switzerland) for three weeks. Her stoma output was reduced from $2800 \mathrm{ml}$ to $400 \mathrm{ml}$ per day by day 10. Serum albumin and serum protein significantly increased also. She subjectively felt better and pain free and stopped her opiate and non- opiate formula. The casein based formula is a nutritionally complete formulation containing a natural antiinflammatory growth factor, transforming growth factor $\alpha 2$. The mechanism for inducing remission in our patient was possibly inhibition of expression of MHC class II protein in downregulating the inflammatory response. ${ }^{2}$

Previous studies have shown that there is a decrease in plasma antioxidant defences in all types of inflammatory bowel disease. ${ }^{3}$ This is mirrored by an increase in free radical peripheral leucocyte DNA damage. It is therefore possible that the casein based formula acts as an antioxidant to minimise the oxidative stress that occurs in patients with active Crohn's disease. Another possible mechanism is that this formula may have a role as a prebiotic by stimulating the activity of bacteria which are already present in the gut.

Remission induced in our case study highlights the part played by a casein based formula in the management of adult Crohn's disease. The encouraging result demonstrates the need to treat similar cases with dietary measures first. This opportunity should not be missed as it may well obviate the need for surgical intervention or administration of potent pharmacotherapeutic agents which carries the risk of several comorbidities.

N Y Haboubi, $S$ Jones

Nevill Hall Hospital, Gwent, South Wales, UK

Correspondence to: $\operatorname{Dr} N$ Y Haboubi, Nevill Hall Hospital, Brecon Rd, Abergavenny, Gwent NP7 7EG, South Wales, UK; nadim.haboubi@ gwent.wales.nhs.uk

Competing Interests: None declared.

\section{References}

1 Jones S, Shannon H, Srivastava E, et al. A novel approach to a patient with Crohn's disease and a high stoma output: a missed opportunity? Scand J Gastroenterol 2004;4:398-400.

2 Donnet-Hughes A, Duc N, Serrant P, et al. Bioactive molecules in milk and their role in health and disease: the role of transforming growth factor- $\alpha$. Immunol Cell Biol 2000:34:49-53.

3 D'Odorico A, BortolanS, Gaardin R, et al. Reduced plasma antioxidant concentrations and increased oxidative DNA damage in inflammatory bowel disease.

Scand J Gastroenterol 2001;36:1289-94.

\section{Identification of ferroportin disease in the Indian subcontinent}

Haemochromatosis is a common inherited disorder of iron metabolism, characterised by excessive iron absorption and deposition in tissues. The majority of cases are associated with mutations in the HFE gene and inherited in an autosomal recessive manner.' Autosomal dominant forms of haemochromatosis have been reported, mainly associated with mutations in the ferroportin 1 gene. ${ }^{2}$ This syndrome, termed type 4 haemochromatosis or more recently ferroportin disease, ${ }^{3}$ is usually characterised by an early increase in serum ferritin with normal transferrin saturation. Iron accumulation is most prominent in Kupffer cells and other macrophages, in addition to hepatocytes. Some patients do not tolerate venesection therapy well and can develop anaemia. Hereditary iron overload disorders appear to be uncommon in Asia. Secondary iron overload due to beta thalassaemia is relatively common in the Indian subcontinent. However, primary iron overload disorders and HFE mutations appear to be rare and cases have not been well characterised in this region. ${ }^{45}$ We identified a patient from the Indian subcontinent with features typical of ferroportin disease.

A 36 year old female of Sri Lankan origin presented for a routine medical examination in December 2003. She was found to have an elevated serum ferritin of $3145 \mu \mathrm{g} / \mathrm{l}$. Her serum iron $(17.1 \mu \mathrm{mol} / \mathrm{l})$ and transferrin saturation $(29 \%)$ were normal. Liver functions tests, blood glucose, and thyroid studies were all normal. Physical examination was normal and she had no significant past medical history or risk factors for iron overload.

C282Y, H63D, and S65C HFE gene mutations were all negative and she had no family history of iron overload. Her mother and three siblings all had normal serum ferritin levels. Her father died of ischaemic heart disease aged 48 years.

A magnetic resonance imaging scan showed hepatic iron overload. Liver biopsy showed grade 3-4 iron deposition within hepatocytes and Kupffer cells; no fibrosis or cirrhosis was evident (fig 1). The hepatic iron concentration was $17700 \mu \mathrm{g} / \mathrm{g}$ dry weight and hepatic iron index was 9.1.

Venesection therapy was initially poorly tolerated with the development of anaemia following the first two $500 \mathrm{ml}$ venesections. Her haemoglobin is now stable on a programme of $300-500 \mathrm{ml}$ venesections every three weeks.

The features of ferroportin disease in this patient led us to sequence the ferroportin 1 gene, as previously described. ${ }^{6}$ Analysis of the DNA sequence revealed a heterozygous three base pair deletion (TTG) in exon 5. This is the same deletion, Vl62del, described by us and others in haemochromatosis patients from Australia, the UK, Italy, and Greece..$^{6-9}$

This is the first report to identify Vl62del or indeed any ferroportin 1 mutation in an individual from the Indian subcontinent. Identification of V162del in an Asian patient confirms that this mutation is likely to be the most common mutation of ferroportin 1 and the most common cause of non-HFE associated haemochromatosis. The wide geographical distribution of this mutation suggests that it is a recurrent mutation that has repeatedly arisen in distinct populations, probably by slippage mispairing.

Iron overload in this patient was typical of ferroportin disease. At the time of diagnosis she was asymptomatic and had no fibrosis on liver biopsy. Whether fibrosis or clinical complications will develop with age if iron stores are not depleted is unclear.

In conclusion, we have identified the V162del mutation of ferroportin 1 in a fifth geographical location, emphasising that this mutation is the most common and widely distributed mutation which causes non-HFE haemochromatosis. We have identified V162del in a region where iron overload disorders have not been well characterised. Analysis of this and other ferroportin 1 mutations may be useful in the study of iron overload disorders in this region and may be the basis of hitherto unexplained cases of iron overload. 

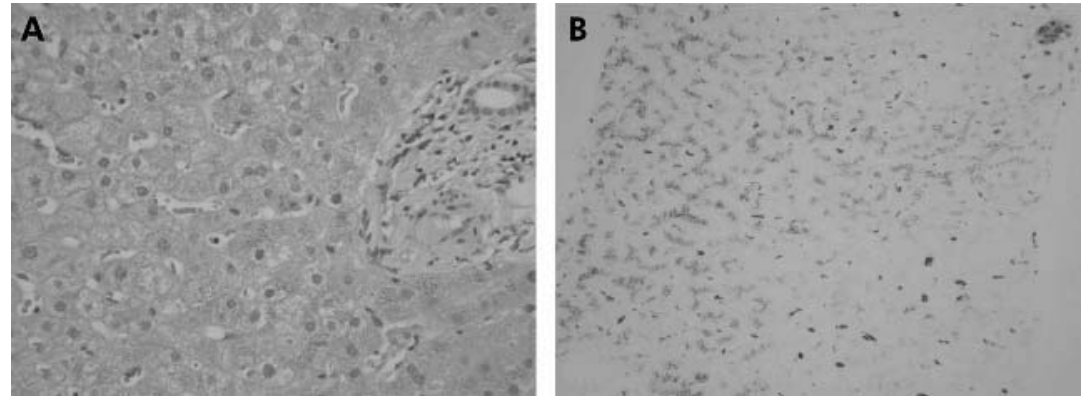

Figure 1 Liver biopsy sections from our patient stained with (A) haematoxylin and eosin and (B) Perls' Prussian blue (magnification 100×). Grade 3-4 iron is prominent in hepatocytes and Kupffer cells.

\section{Acknowledgements}

This work was supported in part by grants from the National Health and Medical Research Council of Australia (953219), the National Institutes of Health, USA (5R01DK057648-02), and the Haemochromatosis Society of Australia to VNS.

D F Wallace

Membrane Transport Laboratory, The Queensland Institute of Medical Research, Brisbane, Queensland, Australia

P Browett

Department of Molecular Medicine and Pathology, University of Auckland, and LabPlus, Auckland City Hospital, Auckland, New Zealand

P Wong

Department of Gastroenterology, Auckland City Hospital, Auckland, New Zealand

H Kua

Diagnostic Medlab, Auckland, New Zealand

R Ameratunga

LabPlus, Auckland City Hospital, Auckland, New Zealand

V N Subramaniam Membrane Transport Laboratory, the Queensland Institute of Medical Research, Brisbane, Queensland,

Australia

Correspondence to: Dr V N Subramaniam, Membrane Transport Laboratory, The Queensland Institute of Medical Research, 300 Herston Rd Herston, Brisbane, QLD 4006, Australia; nathanS@ qimr.edu.au

doi: 10.1136/gut.2004.060988

Competing Interests: None declared.

\section{References}

1 Feder JN, Gnirke A, Thomas W, et al. A novel MHC class I-like gene is mutated in patients with hereditary haemochromatosis. Nat Genet 1996:13:399-408.

2 Pietrangelo A. Non-HFE hemochromatosis. Hepatology 2004;39:21-9.

3 Pietrangelo A. The ferroportin disease. Blood Cells Mol Dis 2004;32:131-8.

4 Kaur G, Rapthap CC, Xavier M, et al. Distribution of C282Y and H63D mutations in the HFE gene in healthy Asian Indians and patients with thalassaemia major. Natl Med J India 2003:16:309-10.

5 Thakur V, Guptan RC, Hashmi AZ, et al. Absence of hemochromatosis associated Cys282Tyr HFE chronic liver disease patients in India. J Gastroenterol Hepatol 2004; 19:86-90.

6 Wallace DF, Pedersen P, Dixon JL, et al. Novel mutation in ferroportin 1 is associated with autosomal dominant hemochromatosis. Blood 2002; 100:692-4

7 Devalia V, Carter K, Walker AP, et al. Autosomal dominant reticuloendothelial iron overload associated with a 3-base pair deletion in the ferroportin 1 gene (SLC11A3). Blood 2002; 100:695-7.

8 Roetto A, Merryweather-Clarke AT, Daraio F, et al. A valine deletion of ferroportin 1: a common mutation in hemochromastosis type 4 . Blood 2002;100:733-4.

9 Cazzola M, Cremonesi L, Papaioannou M, et al. Genetic hyperferritinaemia and reticuloendothelial iron overload associated with a three base pair deletion in the coding region of the ferroportin gene (SLC 1 1A3). Br J Haematol 2002;119:539-46.

\section{BOOK REVIEW}

\section{Morson and Dawson's Gastrointestinal Pathology, 4th edn}

Edited by D W Day, J R Jass, A B Price, et al. $£ 175.00$, pp 692. ISBN 0-632-04204-4

Why do people buy s book such as this, which involves a not inconsiderable financial outlay (even if you box clever and make it tax firstly, for use as a bench book, and secondly, for information on the pathological basis of gastrointestinal disease for interest, teaching, or indeed research purposes.

On the first criterion, this book succeeds, usually quite brilliantly. As a vade mecum on gastrointestinal pathology it should be on the shelf of every pathologist who engages in the reporting of such material. In my view, the book is more user friendly than the gene mutation and low frequency of hemochromatosis phenotype in nonalcoholic Massachusetts: Blackwell Publishing, 2003, deductible)? I think for two main reasons-
competition-Fenoglio-Preiser and Goldman to name but two-and is certainly more readable. I would therefore extol its virtues unreservedly in this respect.

On the second criterion, as a source book, I suppose the correct word is patchy. Some sections, for example that on colorectal tumours, is admirable in this respect, whereas other sections are more limited in scope and even cursory in their treatment of the pathobiology. There is also the problem of the unavoidable intrinsic delay in producing such a book, resulting in reference lists which are some years away from the publication date. I am aware however that my personal outlook is not that of most individuals who will purchase this volume so I am probably being over critical. It is, after all, quintessentially a bench book, and excellent at that.

However, I do have one real beef. In any multiauthor work there is bound to be variation, but here we are not told which one of the stellar caste were responsible for which section or chapter. Of course we can make informed guesses about the Barrett's or colorectal carcinoma sections, but who did the GIST bit? Because of some (minor) errors in the criteria for the diagnosis of malignancy, I have tried to berate a number of authors who have all denied responsibility, and blamed someone else-usually the author(s) absent at the time. Not good enough.

I have to concede however that the authors have succeeded in producing perhaps the text in gastrointestinal pathology, which is a credit to both themselves and the discipline in the UK. I congratulate them.

N A Wright

\section{CORRECTIONS}

doi: 10.1136/gut.2003.025494corr 1

In the January 2005 issue of Gut, one of the author's names of the paper entitled Human peripheral and gastric lymphocyte responses to Helicobacter pylori NapA and AphC differ in infected and uninfected individuals $(\mathrm{H} \mathrm{J}$ Windle, Y S Ang, V A Morales, R McManus, and D Kelleher. Gut 2005;54:25-32) was cited incorrectly. V A Morales should read V AthieMorales. The journal apologises for this mistake.

\section{doi: 10.1136/gut.2003.026807corr 1}

In the December issue of Gut fig 1 in the paper by AJG Bell et al (Human lymphocyte stimulation with pouchitis flora is greater than with flora from a healthy pouch but is suppressed by metronidazole. Gut 2004;53: 1801-1805) is incorrect. The labels for fig $1 \mathrm{C}$ are inverted; the squares should have been labelled HetNon and the triangles HetPM. The legend is also incorrect because the label for flora grown on agar without metronidazole is HetNon, not HetP as stated. 